\title{
Upregulation of RASAL2 promotes proliferation and metastasis, and is targeted by miR-203 in hepatocellular carcinoma
}

\author{
JIAN-FENG FANG ${ }^{1,2}$, HAI-PING ZHAO ${ }^{1,3}$, ZHENG-FEI WANG $^{1,4}$ and SHU-SEN ZHENG ${ }^{1}$ \\ ${ }^{1}$ Division of Hepatobiliary and Pancreatic Surgery, Department of Surgery,
}

The First Affiliated Hospital of Zhejiang University, Hangzhou, Zhejiang 310003; ${ }^{2}$ Department of Hepatobiliary Surgery, Shaoxing People's Hospital, Shaoxing, Zhejiang 312000; ${ }^{3}$ Department of General Surgery, Fuyang People's Hospital, Fuyang, Zhejiang 311400; ${ }^{4}$ Department of Hepatobiliary Surgery, Quzhou People's Hospital, Quzhou, Zhejiang 324000, P.R. China

Received December 7, 2015; Accepted December 6, 2016

DOI: $10.3892 / \mathrm{mmr} .2017 .6320$

\begin{abstract}
RAS protein activator like 2 (RASAL2) has been reported to be dysregulated in various types of cancer. It has previously been demonstrated that RASAL2 is hypomethylated in hepatocellular carcinoma (HCC). However, the expression pattern of RASAL2 and its potential role in HCC remain to be elucidated. The present study demonstrated that the expression of RASAL2 was upregulated in HCC tissues, compared with in normal liver tissues, by using immunohistochemistry. In addition, Cell Counting Kit-8 assay and invasion assay revealed that knockdown of RASAL2 inhibited the growth and invasion of HCC cells. Western blotting results indicated that the inhibition of RASAL2 reduced the levels of phosphorylated-AKT. Notably, RASAL2 was observed to be a direct target of miR-203 in HCC in luciferase activity assays. Furthermore, overexpression of miR-203 exhibited a similar effect to RASAL2 knockdown in HCC cells. These results indicated that RASAL2 serves a tumor oncogenic role in HCC and may be considered a potential target in HCC.
\end{abstract}

\section{Introduction}

Hepatocellular carcinoma (HCC) is reported to be the fifth most common cancer type and the second leading cause of cancer-associated mortality worldwide $(1,2)$. Risk factors are well characterized and surgical resections well developed; however, the long-term survival rate of patients with HCC is poor. The five-year survival rate following resection is limited to $30-40 \%$ (3). It is therefore of primary concern to clarify the

Correspondence to: Dr Shu-Sen Zheng, Division of Hepatobiliary and Pancreatic Surgery, Department of Surgery, The First Affiliated Hospital of Zhejiang University, 79 Qingchun Road, Hangzhou, Zhejiang 310003, P.R. China

E-mail: shusenzheng@zju.edu.cn

Key words: hepatocellular carcinoma, RAS protein activator like 2, microRNA-203, proliferation, metastasis molecular pathogenesis of HCC and develop novel effective treatment strategies.

RAS protein activator like 2 (RASAL2) is a protein that contains a GTPase activating protein (GAP)-related domain, which is characteristic of GAP proteins. A previous study demonstrated that RASAL2 acts as an epithelial cell transforming-2-interacting protein to mediate mesenchymal-amoeboid transition by regulating rhodopsin activity (4). In addition, Feng et al (5) reported that RASAL2 promotes triple-negative breast cancer (TNBC) progression by activating Ras-related $\mathrm{C} 3$ botulinum toxin substrate 1 (RAC1). RASAL2 binds to and antagonizes RAC1-GAP protein ARHGAP24 to suppress breast cancer invasion. Furthermore, RASAL2 has been reported to be hypomethylated in HCC (6); however, the potential role of RASAL2 in HCC remains to be elucidated.

The present study aimed to research the expression pattern and functional role of RASAL2 in HCC. The present study demonstrated that RASAL2 was markedly upregulated in HCC tissues. Functional assays in HuH-7 and HCC-LM3 cells demonstrated that RASAL2 promoted proliferation and invasion in HCC. In addition, results indicated that RASAL2 was a direct target of microRNA (miRNA/miR)-203 in HCC. The data therefore suggested that RASAL2 is important in HCC progression.

\section{Materials and methods}

Patients and tissue samples. The present study included 70 patients underwent resection of $\mathrm{HCC}$ at the First Affiliated Hospital of Zhejiang University School of Medicine (Hangzhou, China). The tumor and non-tumor tissues were maintained in liquid nitrogen immediately following resection. The inclusion criteria required were as follows: i) Diagnosis of HCC confirmed by pathology; ii) without anticancer treatment and distant metastases before surgery; iii) underwent curative resection for HCC between 2010 and 2015, defined as macroscopically complete removal of the tumor with negative safety margin; and iv) with complete clinicopathologic and follow-up data. The characteristics of patients are presented in Table I. This study was approved by the Ethical Review Committee of the First Affiliated Hospital of Zhejiang University School 
of Medicine (Hangzhou, China) and informed consent was obtained from all patients.

Cell lines and culture. HuH-7 and HCC-LM3 liver cancer cell lines were purchased from the Shanghai Institute of Cell Biology (Shanghai, China). These two cell lines were cultured in Dulbecco's modified Eagle's medium (DMEM) (Gibco; Thermo Fisher Scientific, Inc., Waltham, MA, USA) supplemented with $10 \%$ fetal bovine serum (Gibco, Thermo Fisher Scientific, Inc.) at $37^{\circ} \mathrm{C}$ in an atmosphere containing $5 \% \mathrm{CO}_{2}$.

RNA oligoribonucleotides and transfection. Small interfering RNA (si)-RASAL2 (SI04200945), AllStars negative control (NC) siRNA (SI03650318) and miR-203 (MSY0000264) were purchased from Qiagen GmbH (Hilden, Germany). The transfection was performed using Lipofectamine 2000 (Invitrogen; Thermo Fisher Scientific, Inc.) according to the manufacturer's protocol once cells reached 30-50\% confluence.

Reverse transcription-quantitative polymerase chain reaction $(R T-q P C R)$ and western blotting. Total RNA from the HCC cell lines was isolated using TRIzol reagent (Invitrogen; Thermo Fisher Scientific, Inc.) in accordance with the manufacturer's protocol. Subsequently, cDNA was synthesized and qPCR reactions were performed using the ABI7500 Fast system (Applied Biosystems; Thermo Fisher Scientific, Inc.). Briefly, reverse transcription was performed as follows the total $20 \mu 1$ reaction compound consisted of $10 \mu 1$ 2xmiRNA Reaction Buffer mix, $0.1 \% 2 \mu 1$ BSA, $2 \mu 1$ miRNA PrimeScript RT Enzyme Mix, $1 \mu \mathrm{l}$ RNA and $5 \mu \mathrm{l}$ RNase Free $\mathrm{dH}_{2} \mathrm{O}$. All regents used were purchased from Takara Biotechnology Co., Ltd. (Dalian, China). Next, the reaction compound was then incubated at $37^{\circ} \mathrm{C}$ for $1 \mathrm{~h}$ and then $85^{\circ} \mathrm{C}$ for $5 \mathrm{~min}$. Subsequently 80 ul RNase Free $\mathrm{dH}_{2} \mathrm{O}$ was added to the compound and it was stored at $-80^{\circ} \mathrm{C}$ until use. qPCR was performed using a total $20 \mu 1$ reaction volume consisting of $2 \mu 1$ SYBR Premix Ex TaqTM II (2x), $0.8 \mu \mathrm{l}$ PCR Forward Primer $(10 \mu \mathrm{M}), 0.8 \mu \mathrm{l}$ Uni-miR qPCR Primer $(10 \mu \mathrm{M}), 0.4 \mu 1$ ROX Reference Dye II (50x), $2 \mu 1$ synthesized cDNA and $6 \mu 1 \mathrm{dH}_{2} \mathrm{O}$. All the regents used were purchased from Takara Biotechnology Co., Ltd. The thermocycling conditions were as follows: 40 cycles of denaturation for $15 \mathrm{sec}$ at $95^{\circ} \mathrm{C}$ and extension for $60 \mathrm{sec}$ at $60^{\circ} \mathrm{C}$. GAPDH and U6 served as internal controls. The results were quantified by $2^{-\Delta \triangle C q}$ method (7). The sequences of primers used in the present study were as follows: RASAL2, forward CCAAATGTCAGTGGAAGCCTCTC, reverse CTGTGT TGTCCTGGCTTGGAGA; and GAPDH, forward GTCTCC TCTGACTTCAACAGCG and reverse, ACCACCCTGTTG CTGTAGCCAA. The primers for miR-203 (MS00003766) and U6 (MS00033740) were obtained from Qiagen GmbH.

For western blotting, cells were lysed in radioimmunoprecipitation buffer (Beyotime Institute of Biotechnology, Haimen, China) accompanied by phenylmethane sulfonyl fluoride (Beyotime Institute of Biotechnology). The concentration of each protein was quantified by Pierce BCA Protein Assay kit (23225, Thermo Fisher Scientific, Inc.). For each sample, $30 \mu \mathrm{g}$ protein was separated by $10 \%$ SDS-PAGE. The proteins were then transferred to polyvinylidene membranes, which were blocked with 5\% non-fat milk at room temperature for $1 \mathrm{~h}$. The washing regent used for the western blotting analysis was PBST. The primary antibodies used in the study were as follows: RASAL2 (1:300; ab121578; Abcam, Cambridge, MA, USA), phosphorylated-AKT (p-AKT; 1:3,000; ab81283; Abcam, Cambridge, MA, USA), $\beta$-actin (1:4,000; A5441; Sigma-Aldrich; Merck Millipore, Darmstadt, Germany), Anti-rabbit $\operatorname{IgG}$, horseradish peroxidase (HRP)-conjugated antibody (7074, Cell Signaling Technology) and anti-mouse IgG, HRP-conjugated antibody (7076, Cell Signaling Technology). An enhanced chemiluminescence kit (NCI4106, Huiying Biotec, Shanghai, China) was used to visualize the membranes.

Immunohistochemistry (IHC).Immunostaining was performed on paraffin-embedded specimens as previously described (8). The antibody used for IHC was RASAL2 (1:400; ab121578; Abcam, Cambridge, MA, USA). For evaluation of the staining score, semi-quantitative estimation was applied as previously described (9). Intensity was graded as 0 , no staining; i) weak staining; ii) moderate staining; or iii) strong staining. The abundance of positive cells was graded from 0 to $4(0,<5 \%$ positive cells; i) $5-25 \%$ positive cells; ii) $26-50 \%$ positive cells; iii) $51-75 \%$ positive cells; iv) $>75 \%$ positive cells). The final score was obtained by multiplying the two values and all the cases were grouped as RASAL2-negative (scores between 1 and 6) and RASAL2-positive (scores between 7 and 12).

Cell proliferation and transwell assay. The proliferation of the HuH-7 and HCC-LM3 cells was evaluated using the Cell Counting Kit (CCK)-8 (Dojindo Molecular Technologies, Inc., Kumamoto, Japan). The transfected cells were plated into 96 -well plates at a density of $5 \times 10^{3}$ cells/well. Following incubation with the CCK reagent for $2 \mathrm{~h}$, the optical density of each well was measured with a Thermomax microplate reader (Sunnyvale, CA, USA) at a wavelength of $450 \mathrm{~nm}$. The assays were conducted at three different time points $(24,48$ and $72 \mathrm{~h})$ and the experiments were conducted in triplicate.

For the transwell assay, $5 \times 10^{4}$ transfected cells in serum-free DMEM were seeded into the upper chambers of inserts (Corning Incorporated-Life Sciences, Tewkesbury, MA, USA), which were coated with Matrigel (BD Biosciences, San Jose, CA, USA). DMEM supplemented with $10 \%$ fetal bovine serum was added to the lower chambers. Following incubation for $48 \mathrm{~h}$, the lower surface was stained with crystal violet. The cells were then counted under an inverted microscope with x100 magnification.

Luciferase activity assay. A luciferase reporter assay was conducted using the Dual-Luciferase Reporter Assay system (E2920; Promega Corporation, Madison, WI, USA) according to the manufacturer's protocol. HuH-7 cells were seeded in 24-well plates and were co-transfected with miR-203 mimics or NC, and wild-type or mutant-type RASAL2 3 'untranslated region (UTR) plasmid, which was synthesized and mutated by Genechem Co., Ltd. (Shanghai, China). All the binding sites were mutated. The firefly and Renilla luciferase activities were measured by a dual-luciferase reporter assay (Promega Corporation) following transfection for $48 \mathrm{~h}$.

Statistical analysis. The correlations between RASAL2 levels and clinicopathological data were analyzed using a $\chi^{2}$ test. 
Table I. Correlations between RASAL2 levels and clinicopathological characteristics in hepatocellular carcinoma patients.

\begin{tabular}{|c|c|c|c|c|}
\hline \multirow[b]{2}{*}{ Characteristic } & \multirow[b]{2}{*}{ Patients $(n=70)$} & \multicolumn{2}{|c|}{ RASAL2 levels } & \multirow[b]{2}{*}{ P-value } \\
\hline & & Positive & Negative & \\
\hline Age (years) & & & & 1.000 \\
\hline$\leq 50$ & 28 & 18 & 10 & \\
\hline$>50$ & 42 & 27 & 15 & \\
\hline Gender & & & & 0.828 \\
\hline Male & 55 & 35 & 20 & \\
\hline Female & 15 & 10 & 5 & \\
\hline Tumor number & & & & 0.098 \\
\hline Single & 30 & 16 & 14 & \\
\hline Multiple & 40 & 29 & 11 & \\
\hline Tumor size (cm) & & & & $0.015^{\mathrm{a}}$ \\
\hline$\leq 5$ & 26 & 12 & 14 & \\
\hline$>5$ & 44 & 33 & 11 & \\
\hline Tumor node metastasis stage & & & & 0.212 \\
\hline $\mathrm{I}, \mathrm{II}$ & 35 & 20 & 15 & \\
\hline III & 35 & 25 & 10 & \\
\hline Grade & & & & 0.785 \\
\hline Well, moderate & 49 & 31 & 18 & \\
\hline Poor & 21 & 14 & 7 & \\
\hline$\alpha$-fetoprotein (ng/ml) & & & & 0.174 \\
\hline$\leq 400$ & 21 & 11 & 10 & \\
\hline$>400$ & 49 & 34 & 15 & \\
\hline Portal vein invasion & & & & 0.237 \\
\hline Negative & 50 & 30 & 20 & \\
\hline Positive & 20 & 15 & 5 & \\
\hline
\end{tabular}

$\chi^{2}$ tests were used to analyze the correlation. ${ }^{\mathrm{a}} \mathrm{P}<0.05$ was considered to indicate a statistically significant difference. RASAL2, RAS protein activator like 2 .

The comparisons between groups were analyzed using paired Student's $t$-test. The data is presented as the mean \pm standard deviation. Statistical analyses were performed with SPSS software version 17.0 (SPSS Inc., Chicago, IL, USA) and GraphPad Prism 5 (Graphpad Software, Inc., La Jolla, CA, USA). P<0.05 was considered to indicate a statistically significant difference.

\section{Results}

RASAL2 is upregulated in HCC tissues. In order to explore the expression pattern of RASAL2 in HCC, the present study performed IHC on 70 pairs of HCC tissues. As presented in Fig. 1, the immunoreactivity of RASAL2 was primarily located in the cytoplasm. The results indicated that the levels of RASAL2 were markedly increased in HCC compared with in normal liver tissues. Of all the tissues, 45 of $70(64.3 \%)$ HCC tissues were positive for RASAL2, whereas only 15 of $70(21.4 \%)$ normal liver tissues were positive.

Subsequently, the present study investigated whether the levels of RASAL2 were correlated with clinicopathological parameters in these $70 \mathrm{HCC}$ tissues by applying $\chi^{2}$ test (Table I). The results revealed that the positive RASAL2 expression group exhibited a larger tumor size $(\mathrm{P}=0.015)$. However, no further correlations between RASAL2 levels and other clinicopathological characteristics were observed, including, gender, age, tumor number, grade and $\alpha$-fetoprotein.

RASAL2 knockdown inhibits cell proliferation and metastasis in HCC cells. Chemically synthesized siRNA was used to knockdown RASAL2 in HuH-7 and HCC-LM3 cells. RT-qPCR was applied to detect the knockdown efficiency of si-RASAL2 (Fig. 2A). In order to evaluate the proliferation rate, a CCK- 8 assay was conducted. The results revealed that the growth rate decreased following inhibition of RASAL2 in Huh7 and HCC-LM3 cells (Fig. 2B). To further determine the significance of RASAL2 in HCC, a transwell assay was applied to investigate the effects of RASAL2 on HCC cell invasion. It was observed that RASAL2 knockdown markedly reduced the invasion of HCC cells (Fig. 2C). The phosphoinositide 3 kinase (PI3K)/AKT pathway has been reported to be important in HCC proliferation and metastasis $(10,11)$. The present study hypothesized that RASAL2 may regulate the PI3K/AKT pathway in HCC cells. To verify this, the present study investigated p-AKT levels, since p-AKT is the key protein of the 

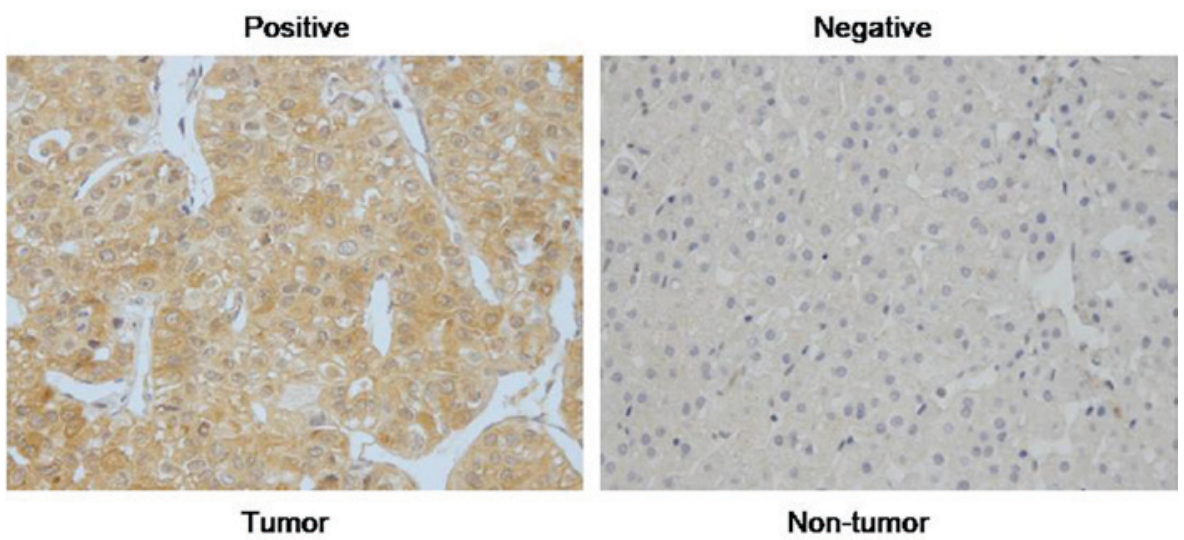

Figure 1. Protein expression of RAS protein activator like 2 was detected by immunohistochemistry. Representative images of tumor and non-tumor tissues were captured at the magnification of $x 400$.

AKT pathway. It was observed that RASAL2 suppression markedly reduced the expression levels of p-AKT compared with the control group in the two cell lines (Fig. 2D). These results indicated that RASAL2 promotes proliferation and invasion in HCC.

RASAL2 is a direct target of miR-203 in HCC. miRNAs have recently been demonstrated to act as critical molecules that regulate proliferation and metastatic progression by binding target genes in HCC (12-14). The present study hypothesized that RASAL2 may be targeted by a specific miRNA in HCC. Following application of TargetScan (http://www.targetscan .org) and miRanda (http://www.microrna.org) computational tools, miR-203 was investigated (Fig. 3A). This specific miRNA was identified by the two bioinformatics tools. Previous studies have reported that miR-203 is downregulated in HCC, and inhibits proliferation and metastasis in $\operatorname{HCC}(15,16)$. The miR-203 mimics were used to enhance the level of miR-203 in HCC cells (Fig. 3A). A dual-luciferase reporter assay was conducted in $\mathrm{HuH}-7$ cells and the results revealed that miR-203 mimics significantly inhibited the luciferase activity of wild-type RASAL2 3'UTR rather than the mutant type (Fig. 3B). In addition, western blotting indicated that the protein levels of RASAL2 were decreased following miR-203 overexpression (Fig. 3B). Furthermore, similar to si-RASAL2, miR-203 mimics exerted anti-proliferative and anti-metastatic effects in HCC cells (Fig. 3C and D). These data suggested that RASAL2 may be a direct target of miR-203 in HCC.

\section{Discussion}

HCC is one of the leading causes of malignancy-associated mortality in humans worldwide. The development of surgical resection, drug targets and other therapeutic strategies is well established; however, the prognosis of $\mathrm{HCC}$ remains poor. The identification of molecular markers in $\mathrm{HCC}$ has been the primary target of research (17-19); therefore, the present study aimed to identify a novel therapeutic biomarker for HCC.

The present study demonstrated that the expression levels of RASAL2 were markedly overexpressed in HCC tissues compared with in normal liver tissues. In a previous study, Stefanska et al (6) reported that the promoters of RASAL2 are hypomethylated, which results in the increased expression of RASAL2 in HCC tissues and cell lines. The data from the present study suggested that RASAL2 acts as an oncogene in HCC development. In addition, the correlation between RASAL2 and clinicopathological features in HCC was investigated, and it was observed that high RASAL2 expression was closely correlated with tumor size. Subsequently, RASAL2 expression was suppressed in HuH-7 and HCC-LM3 cells based on the observations from the HCC tissues, in order to elucidate its functional role in HCC. The results demonstrated that knockdown of RASAL2 decreased the growth rate and metastatic ability of HCC cells. It has previously been reported that RASAL2 functions as a tumor oncogene in TNBC (5), and this is consistent with the results of the present study. The overexpression of RASAL2 in TNBC drives mesenchymal invasion and is correlated with poor outcomes. This further confirms the oncogenic role of RASAL2 in human cancer.

The present study aimed to identify pathways mediating proliferation and metastasis in HCC. The PI3K/AKT pathway, which regulates growth and invasion during cancer development, including HCC, was studied (20,21). Following suppression of RASAL2, the phosphorylation levels of AKT were reduced in the two HCC cell lines. This result suggested that the over-expression of RASAL2 in HCC may activate the AKT pathway resulting in increases in proliferation and invasion.

miRNAs are small non-coding RNAs that regulate gene expression by inhibiting translation and/or stability of mRNA, and thereby contribute to a wide range of physiological and pathological processes (22-24). In human cancer, numerous miRNAs act as potential tumor suppressor genes and low levels of these miRNAs may contribute to overexpression of oncogenic genes $(25,26)$. RASAL2 was revealed to serve an oncogene role in HCC; therefore, the present study hypothesized that high levels of RASAL2 may be attributed to the downregulation of a specific miRNA. miR-203 has been reported to be frequently downregulated in various cancer types, including HCC (27-29). A previous study revealed that RASAL2 is a direct target of miR-203 in breast cancer. The present study, to the best of our knowledge, is the first to confirm that miR-203 directly targets RASAL2 in HCC, according to the following evidence: i) Dual-luciferase reporter assay revealed a decrease in luciferase activity in cells 
A

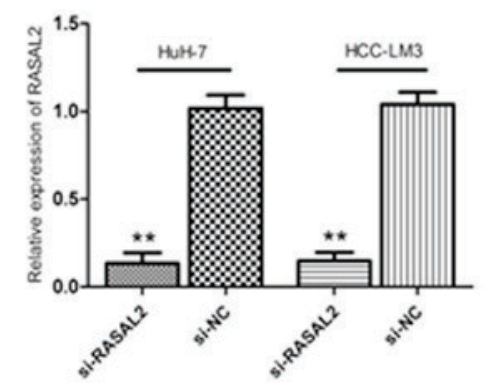

B
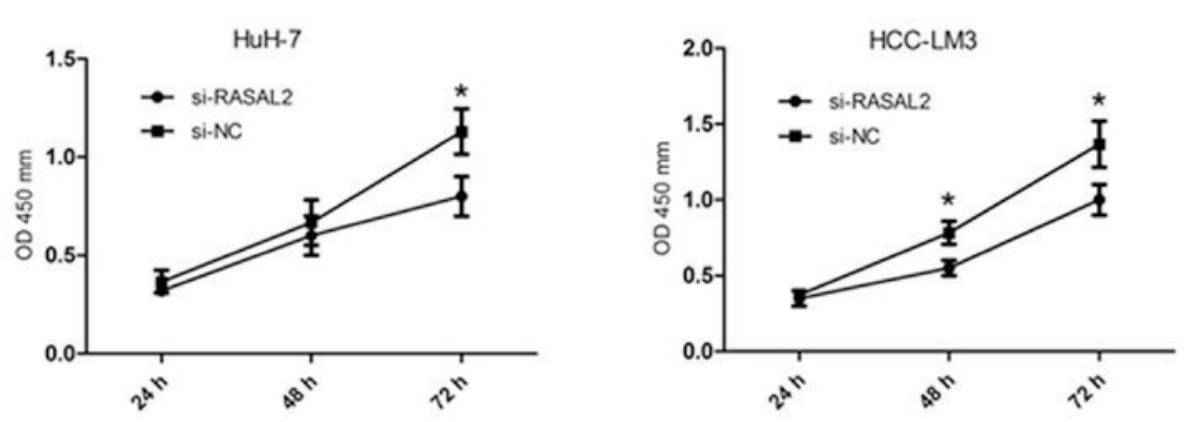

C
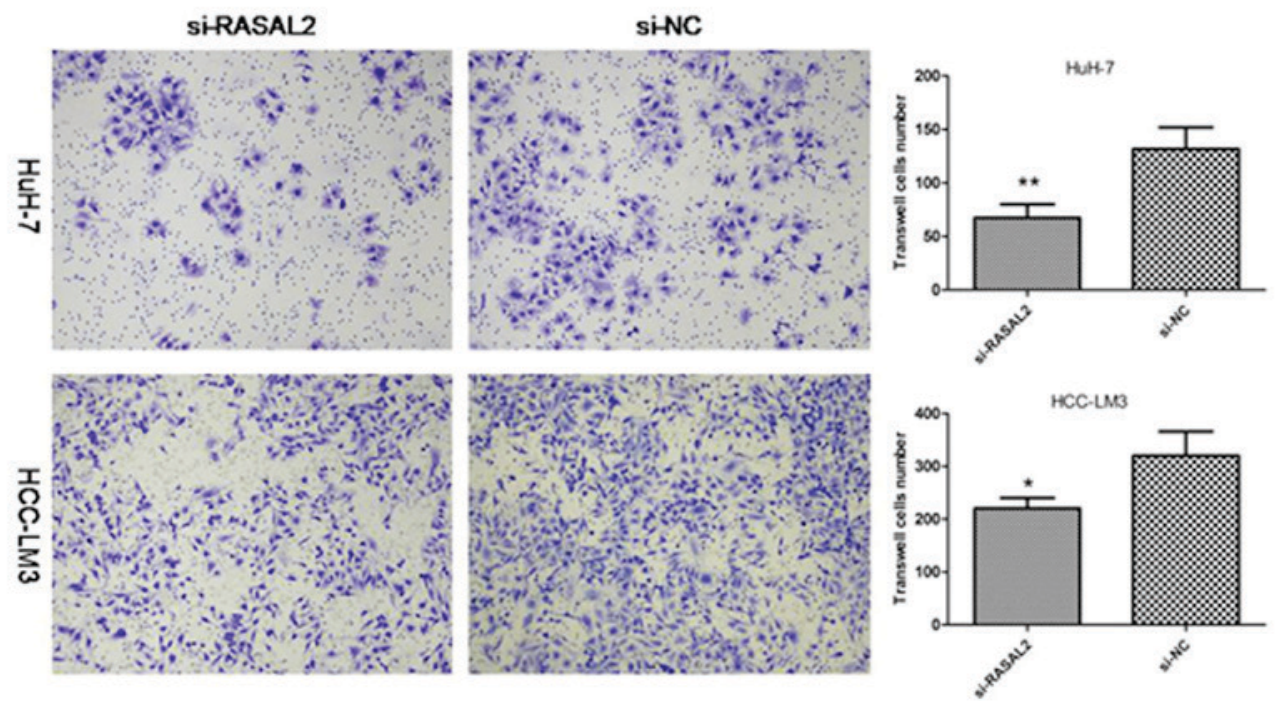

D

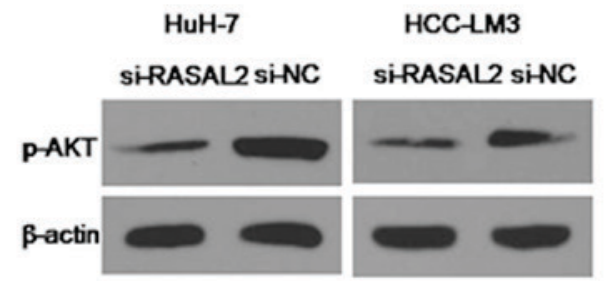

Figure 2. RASAL2 inhibition suppressed cell proliferation and metastasis in HCC cells. (A) Inhibition efficiency of RASAL2 was confirmed by reverse transcription-quantitative polymerase chain reaction. (B) Cell Counting Kit-8 was applied to determine the proliferation rate of HCC cells at three different time points $(24,48$ and $72 \mathrm{~h}$ ). (C) Transwell assay was used to examine the invasive ability of HCC cells. Magnification, x200. (D) Western blotting was used to detect the protein levels of $\mathrm{p}$-AKT following RASAL2 suppression. Representative images are presented. "P<0.05 and ${ }^{* *} \mathrm{P}<0.01$ were considered significant. RASAL2, RAS protein activator like 2; HCC, hepatocellular carcinoma; p-AKT, phosphorylated-AKT; si, small interfering RNA; NC, negative control; OD, optical density. 
A

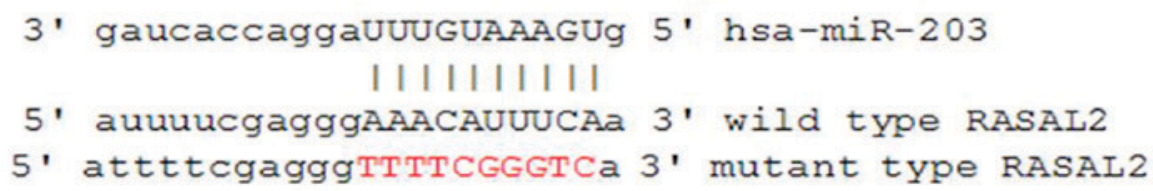

B
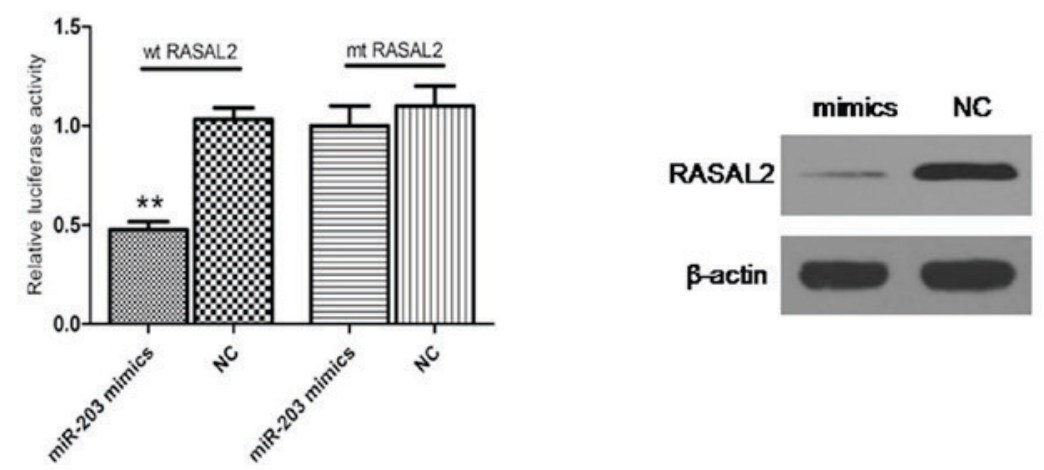

C
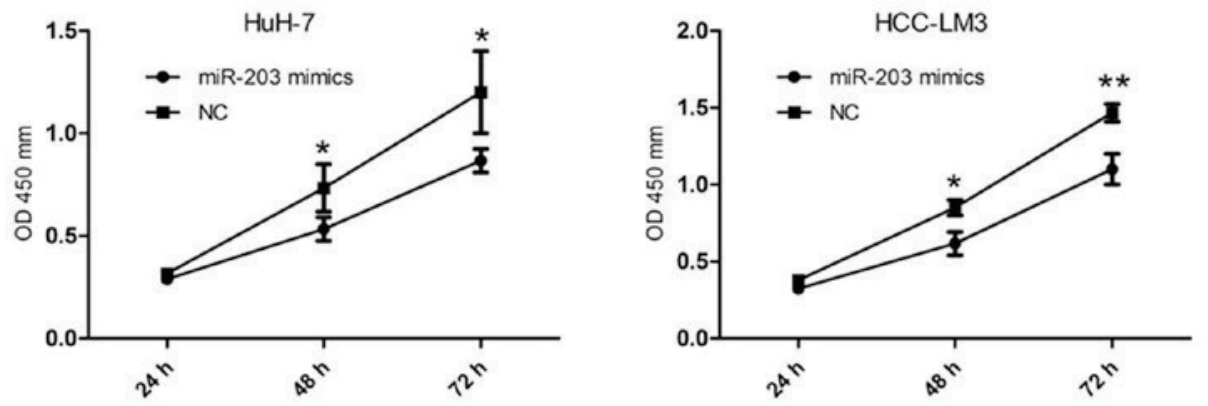

D
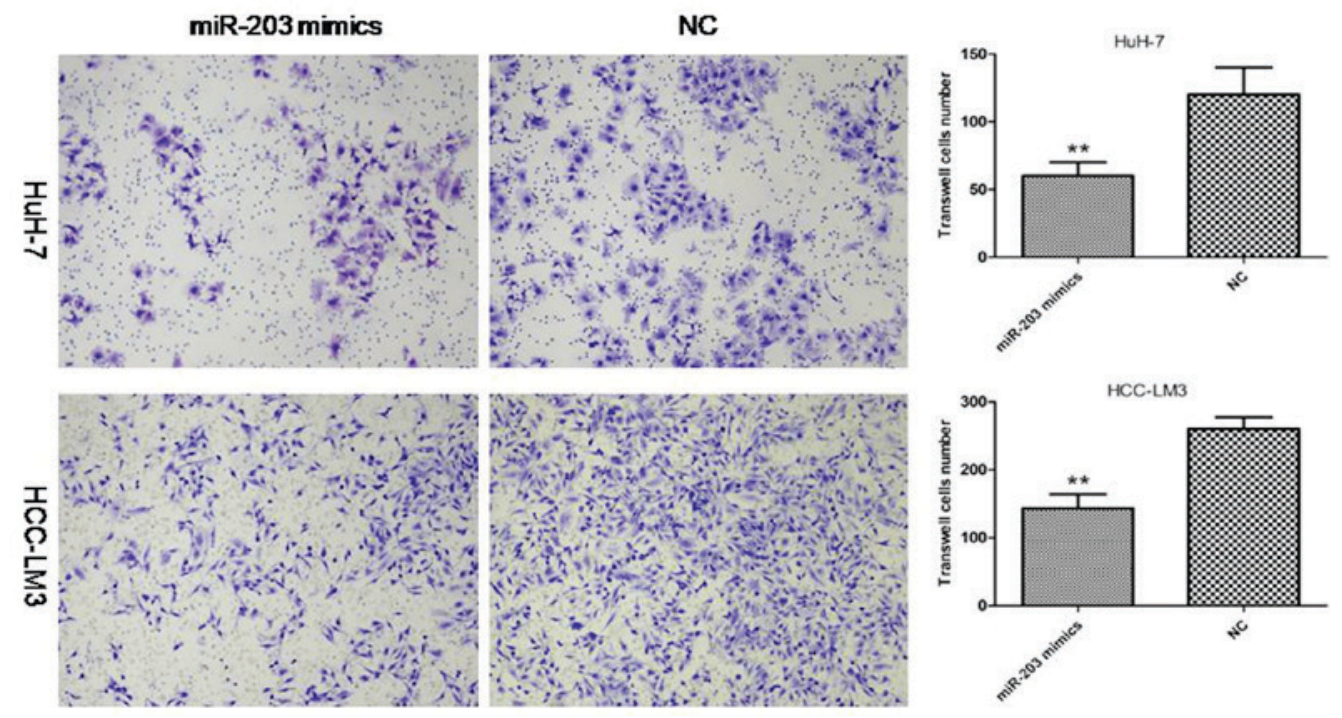

Figure 3. RASAL2 was a direct target of miR-203 in HCC. (A) TargetScan and miRanda predicted the binding sequences of miR-203 and RASAL2, and the complementary sites were mutated. (B) miR-203 mimics reduced the luciferase activity of wt RASAL2 3'untranslated region and protein levels of RASAL2. (C) Cell Counting Kit-8 and (D) Transwell assay indicated that miR-203 mimics inhibited proliferation and metastasis of HCC cells. RASAL2, RAS protein activator like 2; HCC, hepatocellular carcinoma; NC, negative control; wt, wild-type; mt, mutant; miR, microRNA; OD, optical density. ${ }^{*} \mathrm{P}<0.05,{ }^{* *} \mathrm{P}<0.01$.

co-transfected with miR-203 mimics and wild-type RASAL2 3'UTR, whereas no change was observed in cells transfected with the mutant RASAL2 3'UTR; ii) the protein levels of
RASAL2 were decreased following transfection with miR-203 mimics; iii) the effects of miR-203 mimics on proliferation and metastasis were similar to those exhibited following inhibition 
of RASAL2. These data therefore suggested that miR-203 may directly target RASAL2 in HCC.

In conclusion, the results of the present study indicated that RASAL2 is significantly upregulated in HCC tissues. In addition, it was confirmed that RASAL2 promotes proliferation and metastasis by regulating the AKT pathway. Furthermore, it was demonstrated that RASAL2 is the direct target of the tumor suppressor gene miR-203. These data are expected to contribute to mechanistic HCC development and RASAL2 may be considered a potential novel molecular target for the development of future therapeutic technologies.

\section{References}

1. Maluccio M and Covey A: Recent progress in understanding, diagnosing, and treating hepatocellular carcinoma. CA Cancer J Clin 62: 394-399, 2012.

2. Llovet JM, Burroughs A and Bruix J: Hepatocellular carcinoma Lancet 362: 1907-1917, 2003.

3. Altekruse SF, McGlynn KA and Reichman ME: Hepatocellular carcinoma incidence, mortality, and survival trends in the United States from 1975 to 2005. J Clin Oncol 27: 1485-1491, 2009.

4. Weeks A, Okolowsky N, Golbourn B, Ivanchuk S, Smith C and Rutka JT: ECT2 and RASAL2 mediate mesenchymal-amoeboid transition in human astrocytoma cells. Am J Pathol 181: 662-674, 2012

5. Feng M, Bao Y, Li Z, Li J, Gong M, Lam S, Wang J, Marzese DM, Donovan N, Tan EY, et al: RASAL2 activates RAC1 to promote triple-negative breast cancer progression. J Clin Invest 124 5291-5304, 2014

6. Stefanska B, Cheishvili D, Suderman M, Arakelian A, Huang J, Hallett M, Han ZG, Al-Mahtab M, Akbar SM, Khan WA, et al: Genome-wide study of hypomethylated and induced genes in patients with liver cancer unravels novel anticancer targets. Clin Cancer Res 20: 3118-3132, 2014.

7. Livak KJ and Schmittgen TD: Analysis of relative gene expression data using real-time quantitative PCR and the 2(-Delta Delta C(T)) method. Methods 25: 402-408, 2001.

8. Cheng J, Xie HY, Xu X, Wu J, Wei X, Su R, Zhang W, Lv Z, Zheng $S$ and Zhou L: NDRG1 as a biomarker for metastasis, recurrence and of poor prognosis in hepatocellular carcinoma Cancer Lett 310: 35-45, 2011.

9. Noske A, Denkert C, Schober H, Sers C, Zhumabayeva B, Weichert W, Dietel M and Wiechen K: Loss of Gelsolin expression in human ovarian carcinomas. Eur J Cancer 41: 461-469, 2005.

10. Bleeker FE, Felicioni L, Buttitta F, Lamba S, Cardone L, Rodolfo M, Scarpa A, Leenstra S, Frattini M, Barbareschi M, et al: AKT1 (E17K) in human solid tumours. Oncogene 27: 5648-5650, 2008.

11. Sahin F, Kannangai R, Adegbola O, Wang J, Su G and Torbenson M: mTOR and P70 S6 kinase expression in primary liver neoplasms. Clin Cancer Res 10: 8421-8425, 2004.

12. Ohta K, Hoshino H, Wang J, Ono S, Iida Y, Hata K, Huang SK, Colquhoun S and Hoon DS: MicroRNA-93 activates c-Met/PI3K/Akt pathway activity in hepatocellular carcinoma by directly inhibiting PTEN and CDKN1A. Oncotarget 6: 3211-3224, 2015
13. Zhang Z, Zhang Y, Sun XX, Ma X and Chen ZN: microRNA-146a inhibits cancer metastasis by downregulating VEGF through dual pathways in hepatocellular carcinoma. Mol Cancer 14: 5, 2015.

14. Yunqiao L, Vanke H, Jun X and Tangmeng G: MicroRNA-206, down-regulated in hepatocellular carcinoma, suppresses cell proliferation and promotes apoptosis. Hepatogastroenterology 61: 1302-1307, 2014

15. Wei W, Wanjun L, Hui S, Dongyue C, Xinjun Y and Jisheng Z miR-203 inhibits proliferation of HCC cells by targeting survivin. Cell Biochem Funct 31: 82-85, 2013.

16. Wan D, Shen S, Fu S, Preston B, Brandon C, He S, Shen C, Wu J, Wang S, Xie W, et al: miR-203 suppresses the proliferation and metastasis of hepatocellular carcinoma by targeting oncogene ADAM9 and oncogenic long non-coding RNA HULC. Anticancer Agents Med Chem 16: 414-423, 2016.

17. Minguez B and Lachenmayer A: Diagnostic and prognostic molecular markers in hepatocellular carcinoma. Dis Markers 31: 181-190, 2011.

18. Mann CD, Neal CP, Garcea G, Manson MM, Dennison AR and Berry DP: Prognostic molecular markers in hepatocellular carcinoma: A systematic review. Eur J Cancer 43: 979-992, 2007.

19. Forner A, Llovet JM and Bruix J: Hepatocellular carcinoma. Lancet 379: 1245-1255, 2012

20. Wang N, Yao M, Xu J, Quan Y, Zhang K, Yang R and Gao WQ: Autocrine activation of CHRM3 promotes prostate cancer growth and castration resistance via CaM/CaMKK-mediated phosphorylation of Akt. Clin Cancer Res 21: 4676-4685, 2015.

21. Tang B, Qi G, Tang F, Yuan S, Wang Z, Liang X, Li B, Yu S, Liu J, Huang Q, et al: JARID1B promotes metastasis and epithelial-mesenchymal transition via PTEN/AKT signaling in hepatocellular carcinoma cells. Oncotarget 6: 12723-12739, 2015.

22. Bartel DP: MicroRNAs: Target recognition and regulatory functions. Cell 136: 215-233, 2009.

23. Garzon R, Calin GA and Croce CM: MicroRNAs in cancer. Annu Rev Med 60: 167-179, 2009.

24. Di Leva $\mathrm{G}$ and Croce CM: miRNA profiling of cancer. Curr Opin Genet Dev 23: 3-11, 2013.

25. Nicoloso MS, Spizzo R, Shimizu M, Rossi S and Calin GA: MicroRNAs-the micro steering wheel of tumour metastases. Nat Rev Cancer 9: 293-302, 2009.

26. Valastyan S, Reinhardt F, Benaich N, Calogrias D, Szász AM, Wang ZC, Brock JE, Richardson AL and Weinberg RA: A pleiotropically acting microRNA, miR-31, inhibits breast cancer metastasis. Cell 137: 1032-1046, 2009.

27. Liao H, Bai Y, Qiu S, Zheng L, Huang L, Liu T, Wang X, Liu Y, $\mathrm{Xu} \mathrm{N}$, Yan X and Guo H: miR-203 downregulation is responsible for chemoresistance in human glioblastoma by promoting epithelial-mesenchymal transition via SNAI2. Oncotarget 6: 8914-8928, 2015

28. Xu M, Gu M, Zhang K, Zhou J, Wang Z and Da J: miR-203 inhibition of renal cancer cell proliferation, migration and invasion by targeting of FGF2. Diagn Pathol 10: 24, 2015.

29. Liu Y, Ren F, Rong M, Luo Y, Dang Y and Chen G: Association between underexpression of microrna-203 and clinicopathological significance in hepatocellular carcinoma tissues. Cancer Cell Int 15: 62, 2015. 